\title{
ASPECTOS MORFOMETRICOS DE LA CHAMBIRA (Astrocaryum chambira) EN AREAS LIBRES Y BOSCOSAS DE JENARO HERRERA - RIO UCAYALI
}

\section{RESUMEN}

El presente trabajo se realizó en la localidad de Jenaro Herrera, provincia de Requena y región L oreto-Perú, ubicada a la margen derecha del río U cayali a 40 55' $S$ y $73^{\circ} 40^{\prime} \mathrm{W}$.

Se realizaron mediciones de diámetro a la altura del pecho (DAP) altura parcial (h), altura total $(\mathrm{H})$ y proyección de hojas, de la especie Chambira A strocaryum chamhira, Burret) en dos ambientes diferentes a campo abierto y bajo cubierta arbórea.

Para todas las variables se determinó el promedio $(\mathrm{x})$ desviación estandar (5), coeficientes de variación (CV).

Los resultados encontrados, demuestran que existen diferencias importantes en todas las variables medidas; las palmeras que crecen a campo abierto presentan un mayor diámetro, menor altura y hojas con menor longitud. Así mismo las hojas que tienen la orientación este-oeste son mayores a aquellos de orientación norte-sur, a causa de la exposición a la luz solar.

Palabras claves Palmeras, Astrocaryum chambira, morfometría, río U cayali, Perú.

\section{INTRODUCCION}

En la amazonía peruana la familia Palmae esta representada por 33 géneros y más de 150 especies (Spichiger et al, 1990), distribuidas en todos los tipos de bosques de la cuenca (K han y Mejía, 1991). Sus productos o subproductos al natural o transformados primariamente son utilizados masivamente en los sectores rurales y urbanos; sin embargo, existen muy pocos estudios sobre esta familia a pesar que en el Perú, en la localidad de Jenaro Herrera se encuentra la mayor diversidad de palmeras por unidad de área en el mundo.

En la Chambira el endospermo inmaduro es comestible y se comercializa en las ciudades de la selva peruana; de los foliolos jóvenes se extraen fibras que se

Facultad de lngeniería Forestal de la Universidad Nacional de la Amazonía Peruana. Pevas 584.

Apartado 120. Iquitos-Perú 
U tilizan para confeccionar hamacas, shicras, redes, etc. de gran aceptación en los mercados artesanales y de uso generalizado en las áreas rurales.

La Chambira es una palmera monocaule, alta, solitaria de 25-37 m de altura total, que crece en terrenos bien drenados. Rara vez se la encuentra en zonas pantanosas 0 bajas, es muy frecuente hallarla en zonas de colinas y terrenos arcillosos, es fácil de reconocer a simple vista, tanto dentro del bosque como en áreas libres por las largas espinas aplanadas y por la forma de las hojas.

\section{MATERIALES Y METODOS}

El estudio se realizó en la localidad de Jenaro Herrera, provincia de Requena región L oreto-Perú, cuyas coordenadas geográficas son 40 55 5 y 730 40' W; está ubicada a la margen derecha del río U cayali a $150 \mathrm{~km}$ aproximadamente de la ciudad de Iquitos.

Según el mapa ecológico del Perú (ONERN, 1976) el área se encuentra dentro de la formación Bosque Húmedo Tropical; con una temperatura promedio anual de $26.50 \mathrm{C}$ y una precipitación promedio anual de $2521 \mathrm{~mm}$. (Spichiger et al 1989).

$\mathrm{L}$ as mediciones en zonas boscosas se realizaron a la altura del $\mathrm{Km}$. 13.5 al 14.5 de la carretera J enaro Herrera-Colonia A ngamos y a campo abierto en pastizales

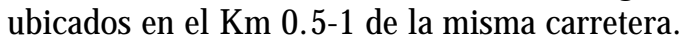

Se consideró a plantas que ya frutificaron midiéndose el diámetro a la altura del pecho (DAP), altura parcial $(\mathrm{h})$, altura total $(\mathrm{H})$ y proyección de bojas.

Para determinar el DAP se usó una forcípula metálica graduada en centímetros, para las alturas se uso una barra telescópica de $15 \mathrm{~m}$, cuando la palma sobrepasaba esta altura se estimaba la diferencia visualmente por segmentos de $5 \mathrm{~m}$; la proyección de hojas se midió con una wincha de tela de 30 $\mathrm{m}$ realizándose 2 medidas una de $\mathrm{N}-\mathrm{S}$ y otras de $\mathrm{E}-\mathrm{O}$.

Para cada variable se determinaron promedio, desviación estándar, coeficiente de variación, distribución de frecuencia y porcentaje en cada rango.

\section{RESULTADOS}

La Tabla 1 muestra las mediciones de las variables observadas para ambos ambientes; asimismo los promedios, desviaciones estándares y coeficiente de variación para estas variables. 


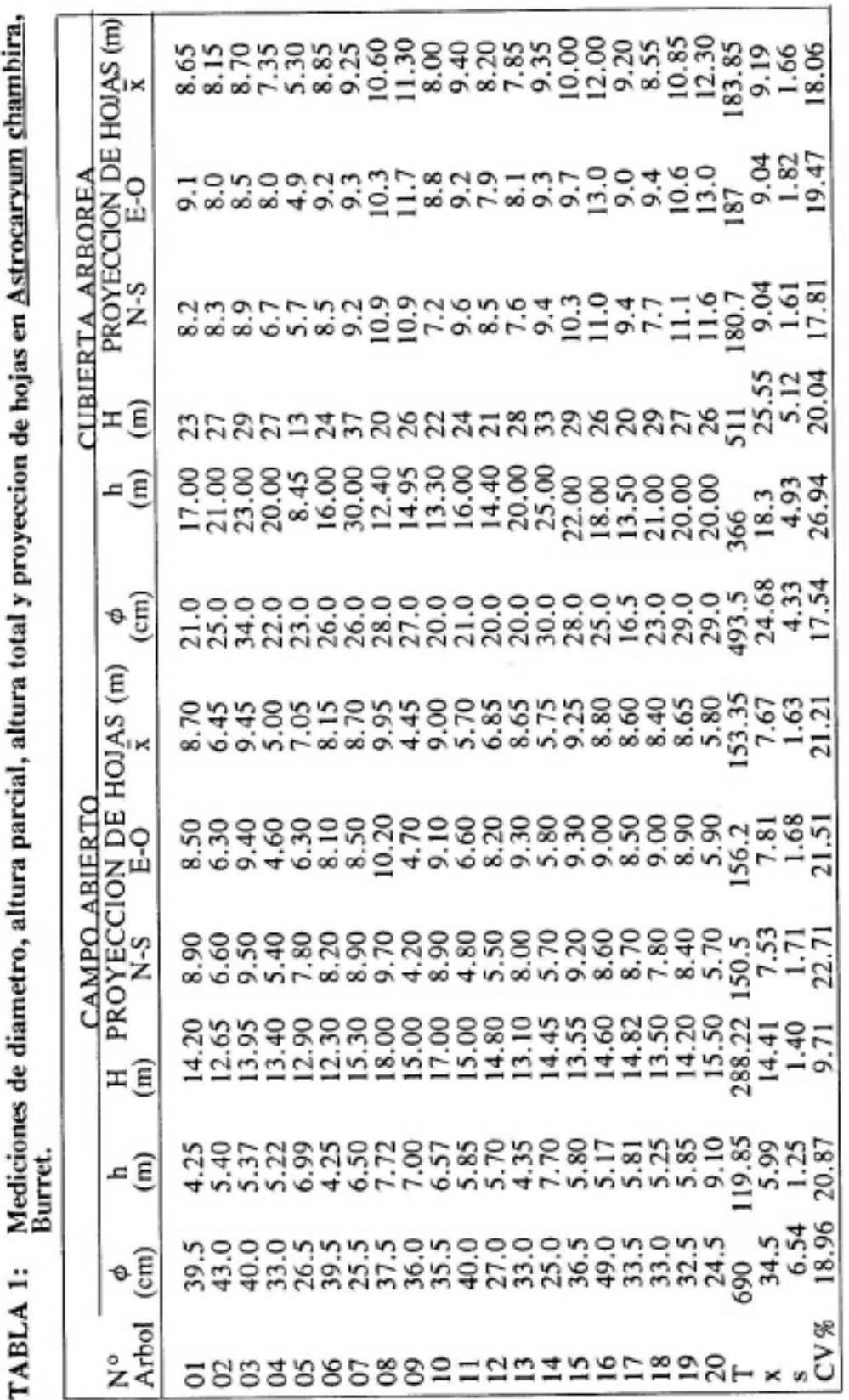


R. ROJAS R.

Las Tablas 2,3,4 y 5 muestran las distribuciones de frecuencia y los porcentajes que ocurren en cada rango de las variables en estudio.

TABLA 2. Frecuencia de Astrocaryum chambira según el diámetro

\begin{tabular}{|c|c|c|c|c|}
\hline \multirow{2}{*}{$\begin{array}{c}? \\
(\mathrm{~cm})\end{array}$} & \multicolumn{2}{|c|}{ CAMPO ABIERTO } & \multicolumn{2}{|c|}{ CUBIERTA ARBOREA } \\
\hline & $\mathrm{N} N$ & $\%$ & $\mathrm{~N} 0$ & $\%$ \\
\hline $15-20$ & - & - & 4 & 20 \\
\hline $20-25$ & 2 & 10 & 7 & 35 \\
\hline $25-30$ & 3 & 15 & 8 & 40 \\
\hline $30-40$ & 5 & 25 & 1 & 5 \\
\hline $35-40$ & 8 & 40 & - & - \\
\hline $40-45$ & 1 & 5 & - & - \\
\hline $45-50$ & 2 & 5 & - & - \\
\hline & 20 & 100 & 20 & 100 \\
\hline
\end{tabular}

TABLA 3. F recuencia de Astrocaryum chambira según la altura parcial.

\begin{tabular}{|c|c|c|c|c|c|}
\hline \multicolumn{3}{|c|}{ CAMPO ABIERTO } & \multicolumn{3}{|c|}{ CUBIERTA ARBOREA } \\
\hline$h(m)$ & $\mathrm{N} \underline{0}$ & $\%$ & $\mathrm{~h}(\mathrm{~m})$ & No & $\%$ \\
\hline $4-5$ & 3 & 15 & $8-9$ & 1 & 5 \\
\hline $5-6$ & 10 & 50 & $9-12$ & - & - \\
\hline $6-7$ & 4 & 20 & $12-13$ & 1 & 5 \\
\hline $7-8$ & 2 & 10 & $13-14$ & 2 & 10 \\
\hline $8-9$ & - & - & $14-15$ & 2 & 10 \\
\hline $9-10$ & 1 & 5 & $15-16$ & 2 & 10 \\
\hline & & & $16-17$ & 1 & 5 \\
\hline & & & $17-18$ & 1 & 5 \\
\hline TOTAL & 20 & 100 & 18-19 & - & - \\
\hline & & & $\begin{array}{l}19-20 \\
20-21\end{array}$ & $\begin{array}{l}4 \\
2\end{array}$ & $\begin{array}{l}20 \\
10\end{array}$ \\
\hline & & & $21-22$ & 1 & 5 \\
\hline & & & $22-23$ & 1 & 5 \\
\hline & & & $23-24$ & - & - \\
\hline & & & $24-25$ & 1 & 5 \\
\hline & & & $25-29$ & - & - \\
\hline & & & $29-30$ & 1 & 5 \\
\hline & & & TOTAL & 20 & 100 \\
\hline
\end{tabular}


TABLA 4. Frecuencia de Astrocaryum chambira según altura total.

\begin{tabular}{|c|c|c|c|c|c|}
\hline \multicolumn{3}{|c|}{ CAMPO ABIERTO } & \multicolumn{3}{|c|}{ CUBIERTA ARBOREA } \\
\hline $\mathrm{h}(\mathrm{m})$ & $\mathrm{N} 0$ & $\%$ & $\mathrm{~h}(\mathrm{~m})$ & $\mathrm{N}$ & $\%$ \\
\hline $12-13$ & 3 & 15 & $13-14$ & 1 & 5 \\
\hline $13-14$ & 5 & 25 & $14-20$ & - & - \\
\hline 14-15 & 8 & 40 & $20-21$ & 3 & 5 \\
\hline $15-16$ & 2 & 10 & $21-22$ & 1 & 15 \\
\hline $16-17$ & 1 & 5 & $22-23$ & 1 & 5 \\
\hline $17-18$ & 1 & 5 & $23-24$ & 2 & 5 \\
\hline TOTAL & 20 & 100 & $24-25$ & - & 10 \\
\hline & & & $\begin{array}{l}23-20 \\
26-27\end{array}$ & $\begin{array}{l}3 \\
3\end{array}$ & 15 \\
\hline & & & $27-28$ & 1 & 15 \\
\hline & & & $28-29$ & 3 & 5 \\
\hline & & & $29-32$ & - & 15 \\
\hline & & & $32-33$ & 1 & - \\
\hline & & & $33-36$ & - & 5 \\
\hline & & & $36-37$ & 1 & 5 \\
\hline & & & TOTAL & 20 & 100 \\
\hline
\end{tabular}

TABLA 5. Frecuencia de Astrocaryum chambira según proyección de las hojas en metros

\begin{tabular}{|c|c|c|c|c|c|}
\hline \multicolumn{3}{|c|}{ CAM PO ABIERTO } & \multicolumn{3}{|c|}{ CUBIERTA ARBOREA } \\
\hline $\mathrm{h}(\mathrm{m})$ & $\mathrm{N} 0$ & $\%$ & $\mathrm{~h}(\mathrm{~m})$ & $\mathrm{N} \underline{0}$ & $\%$ \\
\hline $4-5$ & 2 & 10 & $5-6$ & 1 & 5 \\
\hline $5-6$ & 3 & 15 & $6-7$ & - & - \\
\hline $6-7$ & 2 & 10 & $7-8$ & 3 & 15 \\
\hline $7-8$ & 1 & 5 & $8-9$ & 6 & 30 \\
\hline $8-9$ & 9 & 45 & $9-10$ & 5 & 25 \\
\hline $9-10$ & 3 & 15 & $10-12$ & 2 & 10 \\
\hline TOTAL & 20 & 100 & $12-13$ & 1 & 5 \\
\hline & & & TOTAL & 20 & 100 \\
\hline
\end{tabular}




\section{Diámetros}

A campo abierto el DAP promedio es de $34.5 \mathrm{~cm}$ variando de $24.5 \mathrm{a}$ $49.0 \mathrm{~cm}$ de los cuales el $25 \%$ se encuentran entre 20 y $30 \mathrm{~cm}$ y el $65 \%$ entre $30 \mathrm{y}$ $40 \mathrm{~cm}$, bajo cubierta arbórea el DAP promedio es de $24.7 \mathrm{~cm}$ variando de 16.5 a $34 \mathrm{~cm}$ de los cuales el $20 \%$ se encuentran entre 15 a $20 \mathrm{~cm}$ y el $75 \%$ entre 20 y $30 \mathrm{~cm}$.

\section{Alturas}

A campo abierto la altura parcial (h) promedio es de $6 \mathrm{~m}$ variando de 4.25 a $9.10 \mathrm{~m}$ de los cuales el $15 \%$ están entre 4 y $5 \mathrm{~m}$ y el $70 \%$ entre 5 y $7 \mathrm{~m}$; bajo cubierta arbórea la altura parcial promedio es de $18.3 \mathrm{~m}$ variando de 8.45 a $30 \mathrm{~m}$ de los cuales el $30 \%$ se encuentran entre 13 y $15 \mathrm{~m}$ y otro $30 \%$ entre 19 y $21 \mathrm{~m}$.

Respecto a altura total $(\mathrm{H})$ a campo abierto el promedio es de $14.41 \mathrm{~m}$ con variaciones de 12.30 a $18 \mathrm{~m}$ de los cuales el $15 \%$ se ubican entre 12 y $13 \mathrm{~m}$ y el $65 \%$ entre 13 a $15 \mathrm{~m}$; bajo cubierta la altura promedio es de $25.5 \mathrm{~m}$ con variaciones de 13 a $37 \mathrm{~m}$ de los cuales el $35 \%$ se encuentran entre 20 y $24 \mathrm{~m}$ y el $50 \%$ entre 25 y $29 \mathrm{~m}$.

\section{Proyección de hojas}

A campo abierto el diámetro promedio de proyección es de $7.67 \mathrm{~m}$ con variaciones de 4.45 a $9 \mathrm{~m}$ de los cuales $35 \%$ se encuentran entre 4 y $7 \mathrm{~m}$ y el $60 \%$ entre 8 y $10 \mathrm{~m}$; bajo cubierta la longitud promedio es de $9.19 \mathrm{~m}$ con variaciones de 5.30 a $12.30 \mathrm{~m}$ de los cuales el $70 \%$ se ubican entre 7 y $10 \mathrm{~m}$ y el $20 \%$ entre 10 y $12 \mathrm{~m}$.

La proyección de hojas bajo cubierta es mayor en $1.52 \mathrm{~m}$ en promedio respecto a los que crecen a campo abierto, de igual manera las hojas que están orientadas de $\mathrm{E}$ - $\mathrm{O}$ son mayores a las que están orientadas de $\mathrm{N}$ - $\mathrm{S}$ siendo estas diferencias de 31 y $23 \mathrm{~cm}$ respectivamente para los que están bajo cubierta y a campo abierto.

\section{DISCUSION}

Todas las características morfométricas estudiadas presentan diferencias notables en ambos ambientes, consideramos que se debe fundamentalmente al factor luz y como factor de menor importancia a la competencia arbórea.

Respecto al diámetro y altura, las chambiras que crecen bajo cubierta arbórea son más delgadas y altas que las que crecen a campo abierto, porque estas últimas reciben totalmente la luz solar y no necesitan crecer en longitud para alcanzarla; más bien el crecimiento se realiza en diámetro; mientras que las que están bajo cubierta arbórea crecen en busca de la luz hasta llegar al estrato codominante y en algunos casos al dominante, por eso se explica que el $50 \%$ de ellos se 
encuentran en el rango de 25 a 29 ni que es la altura promedio de los bosques húmedos.

En cuanto a las hojas se observan 2 aspectos importantes; uno el que las hojas de las chambiras que crecen bajo cubierta son más largas que las que crecen a campo abierto, debido también al factor luz y a la competencia del follaje de los árboles circundantes, por cuanto al recibir menos luz tienen que crecer más para captar mayor cantidad de luz para satisfacer sus necesidades, mientras que las que crecen a campo abierto no tienen esta competencia. El otro aspecto es que las hojas que crecen en sentido $E$ - $O$ son mayores que las que crecen de $\mathrm{N}$ - 5, y esto es debido al sentido del movimiento del sol; las hojas se desarrollan más para captar mayor cantidad de luz.

\section{BIBLIOGRAFIA}

KHAN, F.; K. MEJIA, 1991. Las comunidades de Palmeras en los Ecosistemas Forestales inundables de la amazonía peruana. Folia A mazon. $V$ ol 3 4960.

MEJIA, K. (com), 1983. Palmeras y el selvícola amazónico. Lima 8p.

OFICINA NACIONAL DE EVALUACION DE RECURSOS NATURALES (ONERN), 1976. Mapa Ecológico del Perú. Guía Explicativa y Mapa. Lima.

ROJAS, R., 1985. Ensayos de germinación con semillas de 5 especies de Palmeras aplicando 10 tratamientos pre-germinativos y ensayos de cosecha con 7 métodos. Tesis Ingeniero Forestal. U niversidad N acional de la A mazonía Peruana. Iquitos - Perú. 110 p.

SPICHIGER R, R. et al. 1989. Contribución a la flora de la amazonía Peruana. Los árboles del arboretum de Jenaro Herrera. V-I, Genéve. Ed. Conservatoire et J ardin Botaniques. 364 p.

SPICHIGER, R; J. MEROZ, P.A. LOIZEAU; L. STUTZ DE ORTEGA, 1990. Contribución a la flora de la A mazonía Peruana, los árboles del arboretum de Jenaro Herrera. 1976. 146 p. 